\title{
APORTACIONES DEL AUTO DEL TRIBUNAL SUPREMO, DE 11 DE SEPTIEMBRE DE 2009, DE INADMISION DE RECURSO DE CASACIÓN POR INFRACCIÓN DE DISEÑO INDUSTRIAL
}

Lerdys Saray Heredia Sánchez 
El Auto objeto del este comentario fue dictado por el Tribunal Supremo, con fecha 11 de septiembre de 2019, y mediante el mismo se inadmite el recurso de casación interpuesto contra la Sentencia del Tribunal de la Marca de la Unión Europea, con sede en Alicante por infracción de un diseño comunitario. En estas líneas se comenta su contenido, el cual va más allá de un análisis estrictamente procesal pues en los Fundamentos de Derecho encontramos valiosas consideraciones en torno a los factores a tener en cuenta para determinar si existe infracción o no, respecto a la cualidad de usuario informado.

Palabras clave

Diseño comunitario, infracción, usuario informado, reconocimiento judicial, valoración de la prueba.

\section{Abstract}

The judicial resolution object of this comment was issued by the Supreme Court, dated September 11, 2019, and through it the appeal filed against the Judgment of the European Union Trademark Court, based in Alicante by infringement of a community design. These lines content a brief comment about the causes of infringement considered to determine if there is an infraction or not, regarding the quality of the informed user.

Key words

Informed user, community design, infringement, judicial evidence. 


\section{Los hechos sobre los que recae el Auto del Tribunal Supremo y los motivos de casación alegados.}

El Auto objeto del presente comentario se dicta en relación con un recurso extraordinario por infracción procesal y recurso de casación interpuestos por la representación procesal de Société des Produits Nestlé S.A. y Nestlé España S.A. contra la sentencia dictada por la Audiencia Provincial de Alicante (AP), Tribunal de Marca de la Unión Europea (TMUE), Sección Octava, de fecha 18 de noviembre de 2016, en el rollo de apelación 332/C-24/16, dimanante de los autos de juicio ordinario N. ${ }^{\circ} 424 / 2015$, del Juzgado de lo Mercantil $N^{\circ} 2$ de Alicante, de Marca de la Unión Europea al considerar el recurrente, que la STMUE infringe el art. 10.1 del Reglamento 6/2002, de 12 de diciembre de 2001 , sobre dibujos y modelos comunitarios, en relación con los arts. 3, 4.1 y 6 del mismo texto (RDMC), al haber prescindido -a criterio del recurrente- el TMUE de la impresión general que los diseños confrontados producen en los usuarios informados y considerando también que el Tribunal ha infringido el art. 10.1 del RDMC, en relación con los arts. 19.1 y 85.1 del mismo, al haber aplicado criterios propios del enjuiciamiento de las acciones de nulidad del diseño a una acción de violación del mismo. Se trata de diseños aplicados a los envases y cápsulas de café.

La Audiencia estimó el recurso de apelación y revocó la resolución recurrida al no apreciar que se haya producido una infracción del diseño registrado por parte del diseño de la recurrida, ya que la impresión general que causa en un usuario informado es diferente. Ello porque el diseño de un producto de café contiene elementos típicos y comunes, existentes en diversos formatos, lo que determina que los usuarios sean más conscientes de las diferencias entre los distintos productos.
En el primer motivo de casación se denuncia la infracción del art. 10.1 del RDMC, en relación con los arts. 3, 4.1 y 6 del mismo texto, al haber prescindido el TMUE de la impresión general que los diseños confrontados producen en los usuarios informados. El fundamento del motivo se basa en oponerse a la valoración efectuada por el juzgador, en calidad de usuario informado. En este sentido, y a juicio de la parte recurrente, no es posible que el juzgador se arrogue dicha condición respecto de la valoración de la prueba; de forma que no es admisible que se determine que no se ha invadido el ámbito de protección del diseño por un mero reconocimiento judicial, sin haber tenido en cuenta la impresión general que los usuarios informados pueden tener de los diseños confrontados.

En el segundo motivo, se denuncia la infracción del art. 10.1 del RDMC en relación con los arts. 19.1 y 85.1 del mismo, al haber aplicado el TMUE criterios propios del enjuiciamiento de las acciones de nulidad del diseño a una acción de violación del mismo, prescindiendo de las reglas de los apartados 1 de los tres artículos. Según el recurrente, el tribunal debería haberse limitado a decidir de conformidad con lo dispuesto en el art. 10.1 del RDMC, o sea, a examinar si el diseño de la recurrida había invadido el ámbito de exclusión atribuido a la parte titular del diseño, comparando el mismo con los diseños supuestamente infractores para determinar si en los usuarios informados producían o no una impresión distinta.

En el tercer motivo se denuncia la infracción del art. 10.1 del RDMC en relación con los arts. 3 , 4.1 y 6 del mismo texto, al haber prescindido el TMUE de la aplicación de tres reglas: a) la regla de su apartado 1, según la cual la extensión de la protección reconocida a los diseños depende de su carácter singular y b) de la regla de su apartado 1, según la que para determinar esa protección, se tendrá en cuenta el grado de libertad del autor 
al desarrollar el diseño y c) del método a seguir para efectuar la comparación entre los diseños de la actora y la demandada, así como de las consecuencias que de su correcta aplicación se derivan.

En el primer sub motivo (a) se sostiene que la protección reclamada a un diseño no puede ser negada por falta de originalidad, salvo que la acción que hubiera ejercitado el titular se apoyase en normas de propiedad intelectual.

En el segundo sub motivo (b) se defiende que el grado de libertad del autor no se ve afectado por el hecho de que coexistan en el mercado dibujos o modelos similares y que éstos formen una tendencia general y para el recurrente el TMUE se aparta de la norma porque rechazó que una tendencia comercial en materia de diseño pueda considerarse un factor que limite la libertad del autor.

En el tercer sub motivo (c) se manifiesta que no existe una tendencia preexistente al registro que limite la libertad del autor; sino que la tendencia únicamente anticiparía una parte del diseño. El método que emplea el tribunal en su valoración, es analítico, no sintético, a criterio del recurrente.

\section{II.- La valoración del TS.}

Respecto al primer motivo de casación, de acuerdo con el ATS, el recurrente incurre en la causa de inadmisión prevista en el art. 483.2. $2^{\circ}$ LEC relativo a la falta de cumplimiento en el escrito de interposición de los requisitos establecidos para los distintos casos, desarrollada por el Acuerdo del propio TS sobre criterios de admisión de los recursos de casación y extraordinario por infracción procesal de 27 de enero de 2017, por incumplimiento de los requisitos del desarrollo de los motivos, en relación con la falta de respeto a la valoración probatoria, por pretender una revisión de la prueba. La valoración probatoria corresponde al tribunal de instancia, y en el presente caso, la oposición viene dada por el hecho de que se admita que el juzgador se sitúe en la posición de usuario informado.

Tal y como recuerda la sentencia recurrida, la valoración de la prueba supone una valoración conjunta de la materia probatoria obrante en el procedimiento, lo que impide que el criterio de un perito como usuario informado, predomine sobre la propia valoración judicial. En el presente caso, el TMUE tiene en cuenta la clase de diseño en litigio - envases de cápsulas de café- entendiendo que se trata de un diseño referido a un producto de uso común, y por esta razón, el tribunal admite la posibilidad de que sea valorado por el juzgador, en calidad de consumidor común y habitual que también es. $\boldsymbol{Y}$ desde la perspectiva del juzgador, como usuario informado se valora la prueba, respecto de la que muestra su discrepancia la parte recurrente, pero que para el TS carece de razón.

En relación con el segundo motivo alegado, que el tribunal debería haberse limitado a decidir de conformidad con lo dispuesto en el art. 10.1 del RDMC, esto es examinar si el diseño "infractor" había invadido el ámbito de exclusión atribuido a la titular del diseño, comparando el mismo con los infractores para determinar si en los usuarios informados producían o no una impresión distinta, para el TS el recurrente acumula preceptos de contenido heterogéneo en un mismo motivo. En este sentido, entiende que se mezcla el art. 10 RDMC (regulador del ámbito de protección del diseño) con el art. 19 RMDC (que consigna el ius prohibendi) y el art. 85 RMDC, en cuanto a la presunción de validez de los diseños registrados. El resultado de aplicar todos estos artículos es un contenido heterogéneo -lo que apunta el TS con total acierto- puesto que se entrelazan argumentos relativos al grado de libertad del autor, a la valoración del carácter singular o la falta de novedad del diseño, y todo ello no hace más que dar lugar a ambigüedad e indefinición sobre la infracción 
cometida.

El auto comentado declara que la sentencia recurrida no se opone a la doctrina jurisprudencial, ya que la valoración de la singularidad de un diseño depende de las circunstancias fácticas del caso. En este sentido, ha de tenerse en cuenta la jurisprudencia asentada mediante STS 275/2017, de 5 de mayo donde el alto tribunal explica: "Este criterio resulta mucho más adecuado puesto que, como afirmamos en la sentencia 343/2014, de 25 de junio "los ojos que miran" el producto, a efectos de valorar si el diseño goza de singularidad por producir una impresión general diferente a la del diseño registrado, son los del "usuario informado," que presenta un especial cuidado y observa con mayor atención el producto que incorpora el diseño, y no los del simple consumidor medio, normalmente informado y razonablemente atento y perspicaz, que no presta atención a los detalles[...]".

En definitiva, el recurrente incurre en la causa de inadmisión del art. 483.2. $2^{\circ}$ de la LEC, de falta de cumplimiento en el escrito de interposición de los requisitos establecidos para los distintos casos, por incumplimiento de los requisitos del desarrollo de los motivos, en relación con la falta de estructura casacional y acumulación de infracciones.

Conviene recordar que la Sala Esta sala viene diciendo en sus acuerdos y en sus autos (recursos 2412/2016, 4159/2016, 1028/2015) que el escrito de recurso debe estructurarse en motivos, y que tanto si se alega más de una infracción o vulneración de la misma naturaleza como si se alegan varias de distinta naturaleza, cada una de las infracciones debe ser formulada en un motivo distinto y todos ellos deben aparecer numerados correlativamente, sin que puedan formularse sub motivos dentro de cada motivo. Y la parte recurrente incumple estas exigencias, ya que el tercer motivo se divide en tres sub motivos, lo que su- pone que se produzca una acumulación de infracciones en un único motivo, lo que es contrario a la estructura casacional.

\section{III.- Ideas finales.}

En definitiva, para inadmitir el recurso interpuesto, el TS considere acertadamente que la SAP analiza y confronta los diseños en liza y el tipo de producto de que se trata y alcanza la conclusión de que el diseño de la demandada no vulnera el de la demandante y recurrente, ya que como bien apunta el Tribunal en el Auto: "[...]Los diseños de la demandante, aún cuando deban ser considerados válidos por el registro y deba partirse de su singularidad y novedad, se encuentran absolutamente integrados por elementos típicos, evocadores del producto, usuales en otros productos idénticos que hay en el mercado [...] De este modo, el usuario informado no tendrá en consideración esos elementos, o lo ofrecerá poca relevancia, puesto que está habituado a ellos, y considerará, sin embargo, con mayor firmeza las diferencias existentes, para llegar a la conclusión tal y como hace la pericial de la demandada) de que la impresión general es distinta[...]."

Este ATS se convierte en otra oportunidad para recordar a los recurrentes en esta materia que es un error oponerse a la base fáctica de la sentencia al considerar que el tribunal debe limitarse a determinar si la recurrente ha invadido el ámbito de exclusión atribuido a la recurrente y rechaza la posibilidad de que se cuestione el carácter singular. Cuando lo cierto es que la sentencia recurrida sí valora los diseños en atención a sus propias características y finaliza rechazando la infracción del diseño de la recurrente.

También -y desde un punto de vista de técnica procesal- se recuerda que el escrito de recurso debe estructurarse en motivos, y que tanto si 
se alega más de una infracción o vulneración de la misma naturaleza, como si se alegan varias de distinta naturaleza, cada una de las infracciones debe ser formulada en un motivo distinto $y$ todos ellos deben aparecer numerados correlativamente, sin que puedan formularse submotivos dentro de cada motivo (autos relativos a los recursos 2412/2016, 4159/2016, 1028/2015).

Para el TS la sentencia recurrida considera acertadamente que el diseño el litis no vulnera el de la recurrente porque, tras comparar los modelos controvertidos y teniendo en cuenta el grado de libertad del autor, concluye que el diseño de la recurrida produce una impresión general distinta a la producida con los diseños registrados, en el usuario informado. Para ello compara los diseños y tiene en cuenta diversos factores, como por ejemplo, la existencia en el mercado de formatos muy semejantes para la misma clase de productos - por lo que hay poca originalidad-, lo que influye suficientemente para considerar que el usuario informado podrá apreciar con mayor firmeza las diferencias entre los distintos productos en litis.

Para el TS la AP aplicó correctamente las normas, así como la valoración de las pruebas, en consecuencia, no se aprecia que se haya producido una infracción del diseño registrado por parte del diseño de la recurrida, ya que la impresión general que causa en un usuario informado es diferente. Si bien el recurso pudo ser amplio, el recurrente se equivoca, tanto en la forma como en el fondo, y como bien apunta el TS el diseño de un producto de café contiene elementos típicos y comunes, existentes en diversos formatos, lo que determina que los usuarios sean más conscientes de las diferencias entre los distintos productos. Y en consecuencia, recuerda que no ha lugar a la falta de respeto a la valoración probatoria, por pretender una revisión de la prueba. La valoración probatoria corresponde al tribunal de instancia, que admite que el juzgador se sitúe en la posición de usuario informado. 